\section{Pubarca Precoce: Estudo Retrospectivo Clínico e Laboratorial}

\begin{abstract}
RESUMO
Analisaram-se 109 prontuários de pacientes atendidos no período de janeiro de 1990-2000, cujo diagnóstico era pubarca precoce, com o objetivo de se avaliar a etiologia e evolução destes pacientes. Os mesmos foram divididos em 4 grupos: $G_{1}, n=41$ (35F/6M, idades entre 11 meses e 7anos e 11 meses, mediana: 7anos e 1 mês), nos quais foram dosados: 17-OH progesterona (17OHP), DHEA, DHEA-S, androstenediona, testosterona total e/ou livre, e feito teste de estímulo do LH e FSH com GnRH (Relisorm ${ }^{\circledR}, 100 \mu \mathrm{g} i \mathrm{~V}$ ): no grupo $\mathrm{G}_{2}, \mathrm{n}=28(2 \mathrm{~F} / 8 \mathrm{M}$, idades entre 9meses e 8anos e 11 meses, mediana: 7anos e 10meses), além dos exames mencionados, investigou-se a deficiência da 21-hidroxilase através da dosagem de 170HP sob estímulo agudo com ACTH (Cortrosi$n a^{\circledR}, 250 \mu \mathrm{g}$ iv). $O$ grupo $G_{3}, n=23$ (19F/4M, idades entre lano e 2 meses e 8anos e 10meses, mediana: 7anos e 6meses), além dos exames anteriores, foi investigado para deficiências de $11 \beta$-hidroxilase e $3 \beta$-hidroxiesteróide desidrogenase pelas dosagens de 11-deoxicortisol, e relação DHEA/androstenediona, respectivamente. As dosagens de testosterona total e/ou livre. DHEA-S e estradiol (em meninas) foram feitas nos 3 grupos. No grupo $G_{4}, n=14$ (12F/2M, idades entre lano e 1 mês e 8anos e 9meses, mediana: 6anos e 5meses) o diagnóstico foi feito apenas com dados clínicos. Três meninas (6meses, 2anos e 1mês e 2anos e 7meses) foram consideradas à parte; tiveram puberdade precoce associada a hiperandrogenismo, como primeira manifestação de adenocarcinoma adrenal. A investigação demonstrou níveis muito elevados de DHEA-S e massas adrenais ressecadas posteriormente. Um grupo controle de 18 crianças normais pré-púberes $(8 \mathrm{~F} / 1 \mathrm{MM})$ foi submetido ao teste agudo do ACTH. No estímulo com $\mathrm{GnRH}$, um $\Delta \mathrm{LH} \geq 7,0$ foi considerado indicativo de resposta púbere. Andrógenos e estradiol foram dosados por RIE e kits comerciais DPC; LH e FSH foram dosados por métodos imunométricos. As respostas entre pacientes e grupo controle foram comparadas pelo teste de Mann-Whitney. Nos grupos $G_{1}, G_{2} \in G_{3}$ apenas 4 pacientes responderam com um $\Delta \mathrm{LH} \geq 7,0$ e 2 evoluíram com puberdade precoce verdadeira. A resposta da 170HP ao ACTH $\left(G_{2}\right.$ e $\left.G_{3}, n=51\right)$ diagnosticou 2 casos de deficiência da 21-hidroxilase, forma não-clássica. Nos outros, as respostas não diferiram do grupo controle. Não houve acúmulo de outros precursores testados pelo $A C T H$ no $G_{3}$. Concluímos que uma minoria de crianças com adrenarca precoce evoluem para puberdade precoce ou têm hiperplasia adrenal congênita na forma não clássica, o que mostra que, após avaliação dos niveis de esteróides basais, o seguimento apenas clínico seria mais prático e econômico, deixando a realização de testes para quando houvesse algum dado inicial ou evolutivo que $O$ indicasse. (Arq Bras Endocrinol Metab 2000;44/5: 405-412)
\end{abstract}

Unitermos: Pubarca precoce: Adrenarca precoce; Hiperplasia adrenal; Adenocarcinoma adrenal

\footnotetext{
ABSTRACT

The records of 109 patients with premature pubarche, evaluated in the Pediatric Endocrinology Unit during January 1990-2000, were reviewed,
}

artigo original

\author{
Maria F. Borges \\ Fernanda Paula \\ Maria B. Nomeline \\ Fernanda S. Tavares \\ Elvi R. Fonseca \\ Beatriz P. Ferreira \\ Berenice B. Mendonģa
}

Unidade de Endocrinologia Pediátrica da Faculdade de Medicina do Triângulo Mineiro, Uberaba, $M G$ e Laboratório de Hormônios da Universidade de São Paulo, São Paulo, $S P$

Recebido em 27/04/00 Revisado em 28/06/00 Aceito em 11/07/00 
aiming to study the etiology and outcome of this condition. They were divided in 4 groups: group $1, G_{1}$, n: 41, 6 boys, 35 girls, at ages ranging from 11 months to 7.11 years (median: 7.1ys). In this group, basal steroid levels (17-OH-progesterone, DHEA, DHEA-S, $\triangle 4$ - androstenedione, total and free testosterone), $\mathrm{LH}$ and FSH stimulated by acute $\mathrm{GnRH}$ test $(100 \mu \mathrm{g}$ iv, Relisorm ${ }^{\circledR}$ ) were determined. In a second group, $G_{2}$, n: 28,8 boys and 20 girls, at ages between 9 months and 8.1 1ys (median: 7ys), besides $G_{1}$ tests, 21-hydroxylase deficiency (CYP21 $\mathrm{A}_{2}$ ) was investigated by the 17-OH-progesterone response to acute $\mathrm{ACTH}$ test

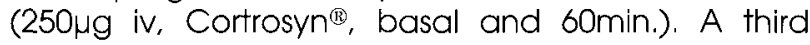
group, $G_{3}, n: 23,4$ boys, 19 girls at ages between $1.2 y s$ and $8.10 y s$ (median: $7.6 y s$ ) underwent the same tests as formers groups, but in addition, the $11 \beta$ -

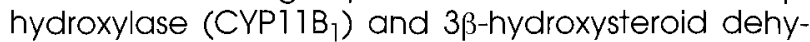
drogenase $(3 \beta \mathrm{HSD})$ deficiencies were ruled out according to 11-deoxycortisol level and the DHEA/ $\triangle 4$-androstenedione ratio respectively, during acute ACTH test. Plasma concentrations of total and free testosterone, DHEA-S and estradiol (in girls) were determined in the 3 groups. A fourth group, $\mathrm{n}: 14,12$ girls and 2 boys, at ages between 1.1y and $8.9 y s$ (median: 6.5ys) had the diagnosis on a clinical basis only. Three girls aged 6 months, 2.1 years and 2.7 years were considered apart, they had premature pubarche and hyperandrogenic signs heralding an adrenal adenocarcinoma. Investigation showed increased DHEA-S level due to adrenal tumor. A control group of 18 pre-pubertal normal children ( 8 girls and 10 boys) underwent an acute ACTH test. During stimulation with $\mathrm{GnRH}$ an increment of $7 \mathrm{mUl} / \mathrm{ml}(\Delta-\mathrm{LH})$ was considered suggestive of pubertal response, Androgens and estradiol levels were measured by RIA (kits from DPC; Los Angeles, California); LH and FSH were measured by immunometric methods. Statistical analysis used Mann-Whitney test. In groups 1, 2 and 3 only 4 patients responded to $\mathrm{GnRH}$ acute test with $\Delta \mathrm{LH} \geq 7 \mathrm{UI} / \mathrm{ml}$ and 2 of them developed true precocious puberty. The 17-OH-progesterone response to acute $A C T H\left(G_{2}\right.$ and $\left.G_{3}, n: 51\right)$ underscored 2 cases of 21-hydroxylase deficiency. In group 3 there wasn't accumulation of other precursors after the acute ACTH stimulation. We observed that very few children with premature adrenarche developed precocious puberty or had congenital adrenal hyperplasia, suggesting that after ruling out adrena tumors, clinical follow-up would be more practical, less expensive and that the tests would be performed when some evolutive clinical finding indicates. (Arq Bras Endocrinol Metab 2000;44/5: 405-412)

Keywords: Precocious pubarche; Precocious adrenarche; Adrenal hyperplasia; Adreno adenocarcinoma

$\mathbf{P}$ UBERDADE PRECOCE CONSISTE no desenvolvimento de qualquer característica sexual secundária antes dos oito anos, nas meninas, e dos nove anos nos meninos. A pubarca precoce é o aparecimento apenas de pêlos pubianos, podendo ser manifestação de amadurecimento do eixo hipotalâmico-hipofisário- gonadal $(\mathrm{HHG})$ e portanto tratar-se de puberdade precoce, ou ser um evento isolado, sem evolução posterior, não obstante maturação precoce da zona reticular do córtex adrenal e produção androgênica (1-6).

Na maioria dos casos de pubarca precoce não se identificam alterações, sendo consideradas variantes da normalidade. Entretanto, alguns autores recomendam que se faça o diagnóstico diferencial com defeitos enzimáticos da esteroidogênese adrenal, principalmente com a deficiência da 21-hidroxilase $\left(\mathrm{CYP} 21 \mathrm{~A}_{2}\right)$ na forma não clássica, a qual pode se manifestar na mulher adulta como acne e hirsutismo e na criança como pubarca precoce. Outros defeitos menos comuns também devem ser afastados, como deficiências de $3 \beta$ hidroxiesteróide desidrogenase (3ßHSD) e $11 \beta$ hidroxilase $\left(\mathrm{CYP} 11 \mathrm{~B}_{1}\right)$ que, embora mais raros, seguem o padrão de manifestações clínicas (7-11).

Enquanto estudos gênicos ainda não estejam disponíveis para a prática clínica, o diagnóstico diferencial, entre pubarca precoce e hiperplasia adrenal congênita, depende da demonstração do acúmulo de esteróides precursores anteriores ao sítio de deficiência enzimática. $\mathrm{Na}$ infância, tais precursores devem ser determinados após estímulo com $\mathrm{ACTH}$, administrado de forma aguda ou crônica, pois os valores basais costumam ser normais. Pela sua maior praticidade, o teste do ACTH agudo foi incorporado como um método útil na investigação laboratorial de rotina da pubarca precoce $(7,12,13)$

No presente estudo propusemo-nos a avaliar clínica e laboratorialmente os casos de pubarca precoce investigados e seguidos na unidade de Endocrinologia Pediátrica da Faculdade de Medicina do Triângulo Mineiro, com o ob̉jetivo de se avaliar a etiologia e evolução destes pacientes, bem como de fazer uma avaliação crítica da utilidade do teste do ACTH agudo na prática ambulatorial.

\section{CASUÍSTICA E MÉTODOS}

Analisaram-se retrospectivamente 109 prontuários de pacientes atendidos no período de janeiro de 1990 a janeiro de 2000 , sendo os mesmos divididos para fins de estudo e de acordo com a investigação efetuada, em quatro grupos:

Grupo 1, $\mathrm{G}_{1}$, n: 41,6 meninos, 35 meninas, com idades entre 11 meses e 7 anos e 11 meses (mediana: 7 anos e 1 mês). Neste grupo, foram dosados esteróides basais: $17-\mathrm{OH}$ progesterona, DHEA, DHEA-S, $\Delta-4$ androstenediona, testosterona total e/ou livre. Não fizeram o teste do ACTH agudo por razões diversas, 
como por exemplo, a falta de Cortrosina ${ }^{\circledR}$ no mercado brasileiro. $\mathrm{O}$ diagnóstico diferencial com puberdade precoce foi feito através do estímulo do LH e FSH com LHRH (Relisorm ${ }^{\circledR} 100 \mu \mathrm{g}$ IV). Nas meninas, ainda foi dosado o estradiol. Além da avaliação laboratorial, a avaliação por imagem incluiu rotineiramente RX de punhos e mãos para determinação da idade óssea, ultrassonografia abdominal e pélvica.

Grupo 2, $\mathrm{G}_{2}, \mathrm{n}: 28$, sendo 8 meninos e 20 meninas, com idades entre 9 meses e 8 anos e 11 meses (mediana: 7 anos e 10 meses). Além dos exames feitos no grupo anterior, neste grupo investigou-se a deficiência da CYP21A $\mathrm{A}_{2}$ através da dosagem de 17$\mathrm{OH}$ progesterona sob estímulo (aos 60 minutos) com ACTH agudo (Cortrosina ${ }^{\circledR}, 250 \mu \mathrm{g}, \mathrm{IV}$ ).

Grupo 3, $\mathrm{G}_{3}$, n: 23, constituído por 4 meninos e 19 meninas, com idades entre 1 ano e 2 meses e 8 anos e 10 meses (mediana: 7anos e 6 meses). Os pacientes deste grupo fizeram todos os exames dos grupos anteriores, mas também foram investigadas as deficiências da CYP11B 1 e 3ßHSD, pelas dosagens do 11 deoxicortisol (Composto S) e a relação DHEA/ $\Delta 4$ androstenediona, respectivamente, durante o estímulo com ACTH agudo aos 60 minutos.

Grupo 4, n: 14 , sendo 2 meninos e 12 meninas, com idades entre 1 ano e 1 mês e 8 anos e 9 meses (mediana: 6 anos e 5 meses) no qual o diagnóstico foi baseado apenas no achado clínico de pubarca precoce. Estas crianças não retornaram para a realização de exames por motivos diversos. Foram posteriormente contatadas e reavaliadas quanto à evolução clínica.

Além destes grupos, 3 meninas com idades de 6 meses, 2 anos e 1 mês e 2 anos e 7 meses apresentaram pubarca precoce como primeira manifestação de adenocarcinoma adrenal.

Um grupo controle de 18 crianças normais pré-púberes, sendo 10 meninos com idades entre 4 anos e 6 meses e 10 anos e 4 meses (mediana: 6 anos e 5 meses) e 8 meninas com idade entre 3 anos e 1 mês e 9 anos e 6 meses (mediana: 7,0 anos) foi submetido ao teste do ACTH agudo com dosagens dos precursores enzimáticos 17-OH-progesterona, Composto $\mathrm{S}$, testosterona, DHEA, DHEA-S, $\Delta 4$ androstenediona e cortisol.

Considerou-se como pubarca precoce o aparecimento de pêlos pubianos com ou sem pêlos axilares antes dos 8 anos nas meninas, sem a presença de telarca, e antes dos 9 anos nos meninos sem a presença de crescimento testicular. Além disso, foram avaliadas a velocidade de crescimento, odor axilar característico, aparecimento de acne, clitoromegalia ou eventual surgimento de mamas durante o seguimento (1-6).
Ultrassonografia abdominal e pélvica (nas meninas), $\mathrm{RX}$ de punho e mão e RX simples de crânio foram feitos empregando-se técnicas convencionais. A determinação de idade óssea foi baseada no método de GrewlichPyle e considerada avançada se houvesse uma diferença maior do que 2 DP da idade óssea esperada $(14,15)$.

Os esteróides $17 \alpha$-hidroxiprogesterona (17$\mathrm{OHP}$ ), dehidroepiandrosterona (DHEA) e seu sulfato (DHEA-S), $\triangle 4$-Androstenediona $(\triangle 4), 11$-deoxicortisol $(\mathrm{S})$, testosterona total (testo) e livre (T-L), estradiol $\left(\mathrm{E}_{2}\right)$ e cortisol (F) foram dosados pelo método de Radioimunoensaio (RIE) usando-se estojos comerciais obtidos da DPC (Diagnostic Products Corporation; LA) (7).

$\mathrm{O}$ teste do estímulo agudo com $\mathrm{ACTH}$ era feito com a criança em posição supina devido a veia puncionada e mantida com soro fisiológico 0,9\%. Após uma hora de repouso era colhido o basal e injetado $250 \mu \mathrm{g} I V$ de Cortrosina ${ }^{\circledR}$ (Laboratório Organon) em bolus. Aos 60 minutos era colhida nova amostra de plasma que, após centrifugada era mantida congelada até a dosagem.

O diagnóstico de deficiência da CYP21 $\mathrm{A}_{2}$ foi considerado se o principal precursor acumulado, $17 \alpha$-OHP, aos 60 minutos, fosse $\geq 10 \mathrm{ng} / \mathrm{ml}$ (7). No caso da deficiência da CYP11B ${ }_{1}$, o Composto $S$, aos 60 minutos pósestímulo deveria ser superior a 3 DP do normal; a deficiência de $3 \beta$-HSD seria cogitada se a relação DHEA $/ \Delta 4$ ou mesmo os valores isolados de DHEA fossem maiores do que 3 DP do normal $(9,13,16,17)$. Entretanto, dados mais precisos como a dosagem de 17-pregnenolona não estavam disponíveis em nosso laboratório.

$\mathrm{Na}$ determinação de LH e FSH empregou-se o método imunofluorimétrico (IFMA) utilizando-se estojos comerciais Delphia de Pharmacia até outubro de 1996 (18). A partir daí, passou-se a utilizar a quimioluminescência automatizada por um sistema IMMULITE, com estojos comerciais de DPC (19).

A indicação ou não de maturidade do eixo HHG foi obtida de acordo com o padrão de resposta

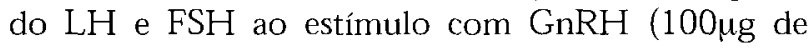
Relisorm ${ }^{\circledR}$, Serono), com dosagens feitas nos tempos basal, 30, 60 e 90 minutos. A resposta do LH e FSH foi avaliada por comparação com um grupo de 18 crianças pré-púberes normais ( 9 meninos e 10 meninas) em estudo previamente publicado, sendo considerada resposta individual positiva, indicativa de maturidade do eixo HHG um $\Delta \mathrm{LH} \geq 7,0 \mathrm{mU} / \mathrm{L}$ (20-23).

O diagnóstico diferencial entre adrenarca precoce e puberdade precoce considerou os dados clínicos, como seguimento de velocidade de crescimento, idade óssea, e um $\Delta \mathrm{LH} \leq 7,0 \mathrm{mU} / \mathrm{L}$ compatível com imaturidade do eixo HHG e, portanto, indicativo de pubarca precoce dentro do contexto clínico (22). 
A comparação dos valores obtidos para cada um dos esteróides no basal e aos 60 minutos pós-estímulo com ACTH, entre o grupo controle e os pacientes investigados, utilizou o teste de Mann-Whitney, e os resultados estão expressos nas tabelas em mediana.

A realização dos testes da cortrosina e do $\mathrm{GnRH}$ em crianças normais (grupo controle) foi aprovada pela Comissão de Ética Médica da Faculdade de Medicina do Triângulo Mineiro e os mesmos foram feitos após obtenção do consentimento informado dos pais.

\section{RESULTADOS}

Os dados clínicos obtidos estão representados na tabela 1, e os dados laboratoriais nas tabelas 2,3 e 4 .

A pubarca precoce foi mais prevalente no sexo feminino (89:20) e embora tenha sido observada entre extremos de idade ( 6 meses a 8 anos e 11 meses) a maioria das crianças tinha entre 6 e 7 anos e 6 meses. (tabela 1)

Dos 109 pacientes, $70(64 \%)$ fizeram retornos regulares a cada 3 meses, sendo que 53 destes já se encontram em idade de desenvolverem puberdade fisiológica, sendo um dado evolutivo importante no diagnóstico; 14 crianças (13\%) não retornaram e não fizeram exames e 25 (23\%) apresentaram acompanhamento irregular. Entretanto, foram contatadas, e segundo informações dos pais, entraram em puberdade em idade esperada, ou permanecem apenas com pubarca precoce.

A maioria das crianças apresentaram estatura, peso e idade óssea dentro dos padrões individuais esperados segundo herança e estado nutricional; 11 tiveram estatura acima de $2 \mathrm{DP}$, enquanto que 23 tiveram desvios negativos; sendo em 5 delas inferior a -2 DP. O peso foi $>2$ DP em 13 crianças; em 21 pacientes observou-se idade óssea avançada, mas sem relação com peso e estatura.

Além de pubarca precoce, $20 \%$ das crianças apresentaram pêlos axilares, 5 meninas tiveram clitoromegalia sendo 1 relacionada ao uso prévio de Danazol ${ }^{\circledR}, 3$ tinham adenocarcinoma adrenal, e 1 com hiperplasia adrenal congênita na forma não clássica por deficiência

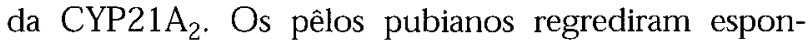
taneamente em uma criança em que a pubarca foi diagnosticada e investigada aos 11 meses $\left(\mathrm{M}_{1} \mathrm{P}_{2}\right)$ e involuiu dentro de 6 meses de observação. Segundo a classificação de Tanner, 15 meninas estavam no estádio $\mathrm{M}_{1} \mathrm{P}_{3}$ e 74 em $\mathrm{M}_{1} \mathrm{P}_{2}$. Os meninos eram $\mathrm{G}_{1} \mathrm{P}_{2}$.

$O$ diagnóstico diferencial entre adrenarca precoce e puberdade precoce foi feito segundo critérios clínicos e pela resposta do $\mathrm{LH}$ ao estímulo do $\mathrm{GnRH}$. Este foi feito em 93 crianças sendo $\Delta \geq$ $7,0 \mathrm{mU} / \mathrm{L}$ em 4. Duas meninas evoluíram para puberdade precoce, com aparecimento de telarca e aumento da velocidade de crescimento. Os outros dois casos foram considerados falsos positivos pois não havia dados clínicos concomitantes indicando maturidade do eixo HHG.

As concentrações basais de estradiol, testosterona total e livre não apresentaram diferença estatística entre pacientes com pubarca precoce e o grupo controle normal, mas o DHEA-S foi significativamente maior nos afetados $(p<0,05)$. (tabela 2)

$O$ estímulo adrenal com ACTH agudo está representado nas tabelas 3 e 4 . A comparação estatística entre o grupo controle e os pacientes com adrenarca demonstrou ser a $\Delta 4-\mathrm{A}$ aos 60 minutos significantemente menor $(p<0,05)$ e, consequentemente, a relação $\mathrm{DHEA} / \Delta 4$ foi mais elevada.

Tabela 1. Dados clínicos do grupo controle e das crianças com pubarca precoce*.

\begin{tabular}{|c|c|c|c|c|c|c|}
\hline Grupo & $\mathrm{n}$ & $M: F$ & $\begin{array}{c}\text { Idade } \\
\text { (anos;meses) }\end{array}$ & DP-Altura & DP-Peso & DP-IO \\
\hline Controle & 18 & $8: 10$ & $\begin{array}{c}6 ; 5 \\
(3 ; 1-10 ; 4)\end{array}$ & $\begin{array}{c}-1,46 \\
(-2,3 / 1,4)\end{array}$ & $\begin{array}{c}-1,11 \\
(-2,1 / 1,0)\end{array}$ & - \\
\hline 1 & 41 & $6: 35$ & $\begin{array}{c}7 ; 1 \\
(11 \mathrm{~m}-7 ; 11)\end{array}$ & $\begin{array}{c}-0,02 \\
(-2,7 / 3,0)\end{array}$ & $\begin{array}{c}-0,04 \\
(-1,6 / 6,0)\end{array}$ & $\begin{array}{c}0,0 \\
(-4,5 / 2,8)\end{array}$ \\
\hline 2 & 28 & $8: 20$ & $\begin{array}{c}7: 10 \\
(9 m-8: 11)\end{array}$ & $\begin{array}{c}0,30 \\
(-1,7 / 4,3)\end{array}$ & $\begin{array}{c}0,05 \\
(-1,1 / 6,3)\end{array}$ & $\begin{array}{c}0,2 \\
(-2,4 / 70,7)\end{array}$ \\
\hline 3 & 23 & $4: 19$ & $\begin{array}{c}7: 6 \\
(1 ; 2-8 ; 10)\end{array}$ & $\begin{array}{c}0,40 \\
(-2,7 / 4,0)\end{array}$ & $\begin{array}{c}0,08 \\
(-1,4 / 5,0)\end{array}$ & $\begin{array}{c}0,7 \\
(-4,4 / 5,0)\end{array}$ \\
\hline 4 & 14 & $2: 12$ & $\begin{array}{c}6: 5 \\
(1 ; 1-8 ; 9)\end{array}$ & $\begin{array}{c}-0,05 \\
(-1,2 / 2,4)\end{array}$ & $\begin{array}{c}-0,18 \\
(-0,9 / 2,8\end{array}$ & $\begin{array}{c}0,10 \\
(-1,3 / 3,3)\end{array}$ \\
\hline
\end{tabular}

* não incluídas 3 meninas com adenocarcinoma adrenal. 
Tabela 2. Dados laboratoriais do grupo controle e dos pacientes portadores de pubarca precoce: basais e resposta do LH e FSH ao GnRH i.v.

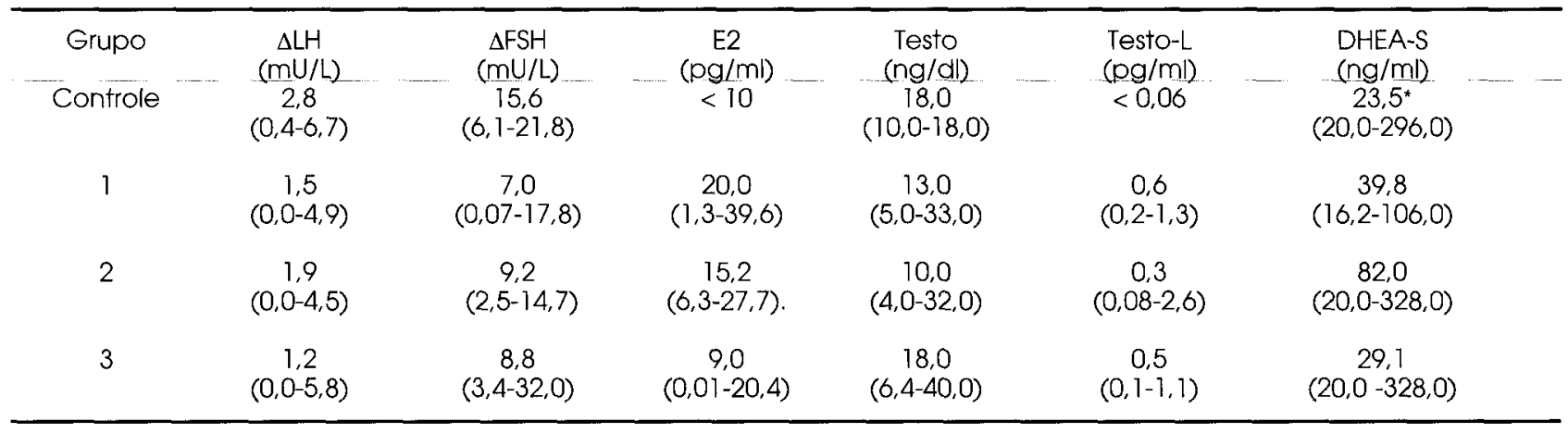

${ }^{*} \mathrm{p}<0,05$

Os critérios individuais para deficiência da CYP21 $\mathrm{A}_{2}$ foram preenchidos por 2 meninas de 7 anos $\mathrm{e}$ 4 meses e 7 anos e 11 meses. A primeira tinha pêlos aumentados com característica de velus generalizado, estava em $\mathrm{M}_{1} \mathrm{P}_{2}$ e apresentava clitoromegalia, idade óssea de 9 anos, DP altura de -1,09 e DP peso de -0,23. O nível basal de $17 \mathrm{OHP}$ de $1,7 \mathrm{ng} / \mathrm{ml}$, sob estímulo com ACTH foi para $20 \mathrm{ng} / \mathrm{ml}$. A segunda menina tinha apenas pubarca, $\mathrm{M}_{1} \mathrm{P}_{2}$, estatura normal, peso excessivo (DP: 1,8) e idade óssea de 11 anos. A 17OHP de $0,56 \mathrm{ng} / \mathrm{ml}$, após estímulo foi para $19,5 \mathrm{ng} / \mathrm{ml}$.

Os critérios para outras deficiências enzimáticas não foram preenchidos por nenhum dos pacientes.

$O$ adenocarcinoma adrenal esteve presente em 3 meninas de 6 meses, 2 anos e 1 mês e 2 anos e 7 meses. $\mathrm{Na}$ primeira, os pêlos pubianos apareceram aos 3 meses e ao exame foi observado acne, clitoromegalia e desvios positivos para peso e altura (1,92 e 1,21 respectivamente). As concentrações de DHEA-S de $252 \mathrm{ng} / \mathrm{ml}$, não supressão do cortisol com dexametasona $(18,4 \mu \mathrm{g} / \mathrm{dl})$ levaram aos exames de imagem, com identificação de adenocarcinoma de $45,7 \mathrm{ml}$ em adrenal esquerda, ressecado posteriormente. A segunda criança tinha pubarca $\mathrm{M}_{1} \mathrm{P}_{3}$, iniciada com 1 ano e 4 meses, clitoromegalia, idade óssea de 3 anos, DP para altura de 1,23 e para peso de 0,81 . Os niveis de DHEA-S de $2720 \mathrm{ng} / \mathrm{ml}$, não supressão do cortisol $(11,8 \mu \mathrm{g} / \mathrm{dl})$, também levaram à identificação de adenocarcinoma de $28 \mathrm{ml}$ na adrenal direita. A terceira menina já chegou com massa abdominal palpável, pubarca precoce e clitoromegalia, mas há 3 meses a mãe havia levado a criança ao pediatra devido a pubarca precoce e havia sido tranqüilizada, sem investigação. Tinha DHEA-S de $5000 \mathrm{ng} / \mathrm{ml}$, cortisol não supressivel $(22,4 \mu \mathrm{g} / \mathrm{dl})$ e adenocarcinoma de $330 \mathrm{ml}$ em adrenal direita.

\section{DISCUSSĀO}

Em 109 casos de pubarca precoce avaliados, encontramos 2 casos de puberdade precoce idiopática, 2 casos de hiperplasia adrenal congênita por deficiência da CYP 21 e 3 casos de adenocarcinoma adrenal, sendo a grande maioria, 102 casos, devido a adrenarca precoce, considerada como variante da normalidade e que não demanda uma forma especial de tratamento, embora estejam surgindo estudos relacionando adrenarca precoce com o desenvolvimento posterior de síndrome dos ovários policísticos, irregularidade menstrual e hirsutismo (11).

Estes dados indicam a heterogeneidade etiológica da pubarca precoce que pode se dever tanto a causas benignas de fácil tratamento, até causas graves como o adenocarcinoma adrenal $(8,9)$. O diagnóstico diferencial e a investigação laboratorial, a priori, de todos os casos, é dispendiosa e pode não estar disponível mesmo no âmbito de um serviço terciário em um hospital universitário, colocando-nos em face do problema de selecionar o nível de investigação mais apropriada e de acordo com o contexto clínico apresentado pelo paciente.

A divisão desta casuística em grupos obedeceu ao nível de investigação conseguida em cada grupo, e a partir da análise dos resultados, alguns critérios de seleção nos parecem mais claros.

Para fazer diagnóstico diferencial de pubarca precoce com puberdade precoce, tendo pêlos pubianos como primeira manifestação, fizemos 90 testes de estímulo do LH e FSH com GnRH. Quatro meninas, que apresentavam apenas pubarca precoce, tiveram um $\Delta \mathrm{LH} \geq 7,0 \mathrm{mU} / \mathrm{L}$, mas somente duas evoluíram com aparecimento de telarca, elevação dos níveis de estradiol, avanço na idade óssea e velocidade 
Tabela 3. Valores dos esteróides basais e após estímulo (60min) com ACTH (Cortrosina ${ }^{(\mathbb{B})} 250 \mu g$ i.v.) nos controles e nos portadores de pubarca precoce.

\begin{tabular}{|c|c|c|c|c|c|c|}
\hline \multirow[t]{2}{*}{ GRUPO } & \multicolumn{2}{|c|}{$\begin{array}{l}\text { Cortisol } \\
(\mu \mathrm{g} / \mathrm{dl})\end{array}$} & \multicolumn{2}{|c|}{$\begin{array}{l}17-\mathrm{OHP} \\
(\mathrm{ng} / \mathrm{ml})\end{array}$} & \multicolumn{2}{|c|}{ Composto S } \\
\hline & 0 & $60 \mathrm{~min}$ & 0 & $60 \mathrm{~min}$ & 0 & $60 \mathrm{~min}$ \\
\hline Controle & $\begin{array}{c}16,0 \\
(7,9-26,5)\end{array}$ & $\begin{array}{c}28,5 \\
(23,4-54,3)\end{array}$ & $\begin{array}{c}0,3 \\
(0,05-1,7)\end{array}$ & $\begin{array}{c}2,2 \\
(0,7-6,1)\end{array}$ & $\begin{array}{c}3,4 \\
(0,0-5,1)\end{array}$ & $\begin{array}{c}5,4 \\
(3,5-6,5)\end{array}$ \\
\hline 2 & $\begin{array}{c}10,0 \\
(5,0-25,0)\end{array}$ & $\begin{array}{c}28,5 \\
(13,2-47,3)\end{array}$ & $\begin{array}{c}0,4 \\
(0,1-1,0)\end{array}$ & $\begin{array}{c}1,3 \\
(0,5-19,5)\end{array}$ & $\begin{array}{c}2,4 \\
(0,9-7,1)\end{array}$ & $\begin{array}{c}4,4 \\
(1,8-9,9)\end{array}$ \\
\hline 3 & $\begin{array}{c}13,3 \\
(5,2-26,0)\end{array}$ & $\begin{array}{c}36,3 \\
(25,2-52,0)\end{array}$ & $\begin{array}{c}0,6 \\
(0,1-0,9)\end{array}$ & $\begin{array}{c}2,9 \\
(0,4-9,5)\end{array}$ & - & - \\
\hline
\end{tabular}

de crescimento, justificando o uso do análogo do $\mathrm{GnRH}$. Assim sendo, julgamos que a avaliação do eixo HHG pelo teste do GnRH não deve fazer parte da avaliação inicial, mas reservado para aquelas crianças cuja evolução clínica demonstre sua necessidade, principalmente pelo aparecimento de telarca precoce.

Nas últimas décadas, numerosos autores relacionaram o aparecimento de pubarca precoce com a presença de defeitos de síntese adrenal em percentuais que variaram de 6 a $45 \%$, de acordo com a existência de uma pubarca típica ou atípica segundo a ausência ou presença de sinais de androgenização (6-9).

Os dados apresentados na literatura sugeriam que, com a finalidade de um diagnóstico etiológico, submetêssemos as crianças com pubarca precoce ao teste do ACTH agudo $(12,13)$ com dosagens dos esteróides adrenais para descartar defeitos das enzimas

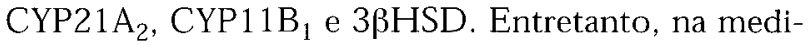
da em que surgiram os estudos gênicos, até mesmo os critérios diagnósticos na interpretação do teste estão sendo questionados (10). No presente estudo, fizemos 51 testes com dosagens de $17 \mathrm{OHP}$ basais e estimulados, o que resultou no diagnóstico de 2 casos (4\%) da deficiência da $\mathrm{CYP}_{21 \mathrm{~A}_{2}}$ na forma não clássica. Analisando retrospectivamente, estes 2 casos eram os que tinham pubarca atípica (9), uma menina com avanço de idade óssea e outra com clitoromegalia associada a hipertricose. Em 23 crianças outros defeitos de síntese foram investigados, e em que pese a falta da dosagem de 17-pregnenolona, não disponível em nosso laboratório, não encontramos defeito da CYP $11 B_{1}$ e da $3 \beta$ HSD. Tratam-se de crianças brasileiras, caucasianas ou mulatas, não tendo entre elas nenhuma de origem judaica, na qual a incidência dos defeitos de síntese é mais prevalente (9).

Os nossos dados indicam a necessidade de seleção das crianças que devem ser submetidas ao teste do ACTH agudo e dosagens estimuladas dos esteróides adrenais e concordamos com Vasconcelos e cols. e outros $(7,9,24)$, que aconselham sua realização em crianças com pubarcas atípicas, com algum nível de androgenização e níveis basais discretamente elevados de $17 \mathrm{OHP}(1,0$ a $3,0 \mathrm{ng} / \mathrm{ml})$. Tal seleção seria menos dispendiosa, mas o controle clínico e seguimento dos pacientes deve ser cuidadoso.

As três crianças com pubarca precoce e adenocarcinoma adrenal não apresentaram dificuldade diagnóstica. O quadro clássico de Cushing estava ausente nas 3 e o que sobressaiu foi o quadro clínico de androgenização em duas, e presença de massa abdominal palpável em uma. $O$ avanço da idade óssea e concentrações séricas de DHEA-S muito elevadas para a idade chamaram a atenção para a presença de tumor adrenal. Entretanto, numa das meninas houve atraso diagnóstico porque o pediatra teve conduta meramente clínica, numa criança com menos de 2 anos, o que nos mostra que é sempre

Tabela 4. Valores dos esteróides basais e após estímulo (60 minutos) com ACTH (Cortrosina@ 250 $\mu g$ i.v.) no grupo controle e no grupo 2 de pacientes portadores de pubarca precoce.

\begin{tabular}{|c|c|c|c|c|c|c|}
\hline \multirow[t]{2}{*}{ Grupo } & \multicolumn{2}{|c|}{$\begin{array}{l}\text { DHEA } \\
(\mathrm{ng} / \mathrm{ml})\end{array}$} & \multicolumn{2}{|c|}{$\begin{array}{c}\Delta 4-\text { Androstenediona } \\
(\mathrm{ng} / \mathrm{ml})\end{array}$} & \multicolumn{2}{|c|}{$\mathrm{DHEA} / \Delta 4$} \\
\hline & 0 & $60 \mathrm{~min}$ & 0 & $60 \mathrm{~min}$ & 0 & $60 \mathrm{~min}$ \\
\hline Controle & $\begin{array}{c}1,6 \\
(0,30-3,90)\end{array}$ & $\begin{array}{c}2,6 \\
(0,90-4,20)\end{array}$ & $\begin{array}{c}0,4 \\
(0,20-2,20)\end{array}$ & $\begin{array}{c}0,3^{*} \\
(0,20-1,20)\end{array}$ & $\begin{array}{c}3,8 \\
(0,20-19,50)\end{array}$ & $\begin{array}{c}8,4^{* *} \\
(2,30-17,00)\end{array}$ \\
\hline 2 & $\begin{array}{c}1,3 \\
(0,01-3,50)\end{array}$ & $\begin{array}{c}2,2 \\
(0,03-6,90)\end{array}$ & $\begin{array}{c}0,2 \\
(0,10-1,00)\end{array}$ & $\begin{array}{c}0,6^{*} \\
(0,20-1,90)\end{array}$ & $\begin{array}{c}4,0 \\
(0,10-10,00)\end{array}$ & $\begin{array}{c}3,3^{* \star} \\
(0,10-19,00)\end{array}$ \\
\hline
\end{tabular}

$* * * * 0,05$ 
mais prudente determinar um perfil androgênico basal em todas as crianças com pubarca precoce.

A adrenarca precoce, como variante da normalidade e causa de pubarca precoce, esteve presente em 102 casos $(93 \%)$, o que nos indica que a maioria destas crianças têm uma causa simples de precocidade sexual, que não demanda tratamento, mas uma conduta expectante e que oriente os pais em como proceder com a criança e principalmente com relação à educação sexual e auxilie a dirimir preocupações como estatura alvo, idade de aparecimento de menarca, após termos descartado as doenças referidas anteriormente. Observamos tendência dos pais de, após tranqüilizados, faltarem aos retornos clínicos e este fato precisa ser discutido, pois o quadro da criança pode mudar se aparecer evolutivamente um dado novo como, por exemplo, telarca.

É evidente, nesta casuística, o predomínio de pubarca precoce no sexo feminino. Não houve diferença significante entre níveis de estradiol e testosterona entre crianças afetadas e grupo controle, ou entre crianças do sexo feminino e masculino neste grupo. As alterações que encontramos foram as já discutidas na literatura, quais sejam níveis significantemente maiores de DHEA-S e $\Delta 4$ (7-9). Balducci e cols. (25) descartaram a existência de alterações em proteínas transportadoras (SHBG) na adrenarca precoce em ambos os sexos, Ruddick e cols. (26) observaram aumento dos níveis de $3 \alpha$ androstenediol como efeito da metabolização aumentada dos andrógenos adrenais, mas em seu grupo controle os níveis não eram diferentes nos meninos em relação às meninas. Não parece, portanto, haver conversão periférica do DHEA-S e $\Delta 4$ em testosterona diferente nas meninas em relação aos meninos, mas é possível que no sexo feminino haja diferença de sensibilidade tecidual aos níveis de andrógenos adrenais, resultando no aparecimento mais freqüente de pêlos axilares e pubianos.

Não obstante a observação de adrenarca precoce em todas as idades, a maioria das crianças estavam entre 6 e 7 anos e 6 meses; sendo que a maioria não tinha idade óssea avançada, desvios significativos no peso e altura, e 53 pacientes já haviam sido seguidos até atingirem puberdade normal em idade adequada. Estes dados indicam a evolução benigna desta condição. Outros autores já demonstraram que a adrenarca precoce não interfere na estatura final ou no início ou ritmo de evolução da puberdade $(27,28)$.

Vários autores têm, ao longo do tempo, tentado explicar os achados clínicos e laboratoriais presentes na adrenarca precoce (16), mas concordamos com Oberfield e cols. (9) de que seria impossível explicar a pubarca precoce naquelas crianças que têm níveis de DHEA-S e $\Delta 4$ normais, a menos que se pense que tais crianças teriam uma resposta tecidual local aumentada ao estímulo androgênico. Esta questão permanece em aberto, à espera de novos estudos.

Os dados obtidos no presente estudo nos levam a concluir que uma minoria de crianças com pubarca precoce evoluem para puberdade precoce ou têm hiperplasia adrenal congênita na forma não-clássica, e que o seguimento clínico após uma primeira avaliação laboratorial dos esteróides adrenais seria o recomendável, deixando a realização de testes para quando houvesse algum dado que o indicasse, como avanço significativo de idade óssea, sinais de hiperandrogenismo ou níveis basais de $17 \mathrm{OHP}$ nos limites superiores da normalidade. Além disso, pubarca precoce em crianças muito pequenas, associada a níveis elevados de DHEA-S são sinais de alarme para o adenocarcinoma adrenal, sendo mandatória a realização de exames de imagem, mesmo na ausência de quadro clínico de síndrome de Cushing.

\section{REFERÊNCIAS}

1. Marshall WA, Tanner JM. Variations in the pattern of pubertal changes in girls. Arch Dis Child 1969:44:291-303.

2. Marshall WA, Tanner JM. Variations in the pattern of pubertal changes in boys. Arch Dis Child 1970;45:13-23.

3. Reiter EO, Grumbach MM. Neuroendocrine control mechanisms and the onset of puberty. Ann Rev Physiol 1982:44:595-613

4. Tanner JM. Sequence and tempo in the somatic changes in puberty. In: Grumbach MM, Grave GD, Mayer FE, eds. Control of the Onset of Puberty. New York:John Wiley \& Sons, 1974;448-70.

5. Colli AS, Crescimento e desenvolvimento pubertário em crianças e adolescentes brasileiros. VI. Maturação sexual. São Paulo:Ed. Bras. de Ciências, 1988.

6. Balzo P, Borrelli P, Cambiaso P, Danielli E, Cappa M. Adrenal steroidogenic defects in children with precocious pubarche. Horm Res 1992;37:180-4.

7. Leite MV, Mendonça BB, Arnhold IJP et al. Investigation of nonclassical 21-hidroxylase deficiency in girls with precocious pubarche. J Endocrinol Invest 1991;14:1 1-5.

8. Siegel SF, Finegold DN, Urban MD, McVie R, Lec PA, Premature pubarche: etiological heterogeneity, J Clin Endocrinol Metab 1992;74:239-47.

9. Oberfield SE, Mayes DM, Levine LS. Adrenal steroidogenic function in a black and Hispanic population with precocious pubarche. J Clin Endocrinol Metab $1990 ; 70: 76-82$.

10. Sakkal-Alkaddour $H$, Zhang $L$, Yang $X$ et al. Studies of $3 b-$ hydroxysteroid dehydrogenase genes in infants and children manifesting premature pubarche and increased adrenocorticotropin-stimulated $D^{5}$-steroid levels. J Clin Endocrinol Metab 1996;81:3961-5. 
11. Lazar L, Kauli R, Bruchis C, Nordenberg J, Gatzer A, Pertzelan A. Early polycystic ovary-like syndrome in girls with central precocious puberty and exaggerated adrenal response. European J Endocrinol 1995; 133:403-6.

12. Toscano V, Balducci R, Adamo MV, Mangiantini A, Cives C, Boscherini B. Changes in steroid pattern following acute and chronic adrenocorticotropin administration in premature adrenarche. J Steroid Biochem 1989;32:321-6.

13. Hawkinse LA, Chasalow C, Blethen SL. The role of adrenocorticotropin testing in evaluating girls with premature adrenarche and hirsutism/oligomenorrhea. J Clin Endocrinol Metab 1992;74:248-53.

14. Greulich WW, Pyle SI. Radiographic Atlas of Skeletal Development of the Hand and Wrist. 2nd ed Stanford:Stanford University Press, 1959

15. Marcondes E. Idade Óssea em Pediatria. In: Marcondes E, editor. Crescimento Normal e Deficiente. $3^{a}$ ed. São Paulo: Sarvier, 1989;33-40.

16. Morris AH, Reiter EO, Geffner ME, Lippe BM, Itamí RM, Mayes DM. Absence of nonclassical adrenal hyperplasia in patients with precocious adrenarche. J Clin Endocrinol Metab 1989:69:709-15.

17. Pang S, Lerner AJ, Stoner E et al. Late-onset adrenal steroid 3b-hydroxysteroid dehydrogenase deficiency I. A cause of hirsutism in pubertal and post-pubertal women. J Clin Endocrinol Metab 1985;60:428-39.

18. Hemmilä I, Dakabu S, Mukkala V-M et al. Europium as label in time-resolved immunofluorimetric assays. Anal Biochem 1984; 137:335-43.

19. Oliveira MC, Neto EC, Pizarro $C B$, Messinger HP Tannhauser M. Valores basais de LH e FSH em crianças e adolescentes normais determinados pelo método da quimioluminescência. Arq Bras Endocrinol Metab 1996:40:198-202.

20. Mendonça BB, Borges MF, Batista MC et al. Comparação entre o método fluorimétrico (FIA) e radioimunoensaio (RIA) na determinação dos níveis de gonadotrofinas basais e após GnRh em crianças normais e pré-púberes. Arq Bras Endocrinol Metab 1992;36(supl.1) :28(abst 23).
21. Borges MF, Mendonça BB, Nomeline MBFL et al. Avaliação da resposta do LH e FSH ao GnRH utilizando-se o método imunofluorimétrico (FIA) em crianças com puberdade precoce verdadeira idiopática. Arq Bras Endocrinol Metab 1995;39(supl. 1):42(abst 32).

22. Borges MF, Nomeline MBFL, Ferreira BP et al. Precocidade Sexual: Estudo retrospectivo clínico e laboratorial. Arq Bras Endocrinol Metab 1998;42:122-202.

23. Britto VN, Batista MC, Borges MF et al. Diagnostic value of fluorimetric assays in the evaluation of precocious puberty. J Clin Endocrinol Metab 1999:84:3539-44.

24. Avivi I, Pollack S, Gideoni O et al, Overdiagnostic of 21hydroxylase late onset congenital adrenal hyperplasia: correlation of corticotrophin test and human leukocyte tipping. Fertility and Sterility 1996;66:557-63.

25. Balducci R, Finocchi $G$, Mangiantini A et al. Lack of correlation between sex hormone binding globulin, adrenal and peripheral androgens in precocious adrenarche. $\mathbf{J}$ Endocrinol Invest 1992; 15:501-5.

26. Riddick LM, Garibaldi LR, Wang ME et al, 3a-Androstanediol glucuronide in premature and normal pubarche. J Clin Endocrinol Metab 1991;72:46-50.

27. Cameron FJ, Tebbutt N, Montalto J et al. Endocrinology an auxology of sibships with non-classical congenital adrenal hyperplasia. Arch Dis Child 1996:74:406-11.

28. Ibañez L, Virdis R, Potau $N$ et al. Natural history of premature pubarche: an auxological study, J Clin Endocrinol Metab 1992:74:254-7.

\section{Endereço para correspondência:}

Maria de Fátima Borges

Disciplina de Endocrinologia, Departamento de Clínica Médica

Hospital Escola da Faculdade de Medicina do Triângulo Mineiro

Rua Getúlio Guaritá s/n

38.025-400 Uberaba, MG 